\title{
XV
}

\section{NOTE SUR IA LANGUE ET L'ECRITURE INCONNUES DES DOCUMENTS STEIN-COWLEY}

PAR R. GAUTHIOT

IE fascicule du mois de janvier $1911 \mathrm{du}$ JRAS. contient un mémoire de M. A. Cowley intitulé "Another Unknown Language from Eastern Turkestan", dont il paraît impossible de dire qu'il n'est pas "sensationnel ". Il nous apporte en effet une écriture inconnue d'origine araméenne, une langue mystérieuse, des documents d'un type précieux et rare puisqu'il s'agit de lettres et non de textes publics religieux ou civils; il nous fournit enfin des textes dont il est possible de déterminer l'âge de façon approximative, dont la haute antiquité est à peu près certaine et qui proviennent du limes chinois lui-même, d'un point situé, il est vrai à l'ouest de Touenhouang mais à l'extrême limite de ce que l'on peut appeler le Turkestan. Pour tous ceux qui s'intéressent à l'histoire de l'Asie, à la résurrection des langues, des peuples, et des civilisations qui ont vécu entre la Chine, la Perse, et l'Inde, que d'attraits nouveaux et de problèmes irritants !

Avec une conscience et un sang-froid des plus remarquables, M. Cowley s'est gardé d'insister dans son travail sur le caractère nouveau, l'aspect imprévu ou l'intérêt actuel des documents qu'il présentait au public pour la première fois. Il s'est attaché, au contraire, à fournir à ceux que les textes rapportés par M. M. A. Stein devaient intéresser des documents sûrs, des résultats critiqués avec soin, des lectures que chacun peut contrôler sur un fac-similé joint à l'article. Il a agi aussi en véritable savant en publiant ce qu'il savait, bien qu'il fût d'avis que c'était peu de chose, en se refusant à garder par devers lui ce que d'autres plus heureux pourraient 
peut-être lire un peu mieux. Et il ne faut pas perdre de vue que les quelques notes et indications qui suivent reposent en entier sur les travaux préliminaires de M. Cowley ; elles se présentent à l'examen et à la critique compétents de la même façon et dans les mềmes conditions que celles de M. Cowley; elles aussi ne visent qu'à être utiles à l'ouvre commune.

Deux points doivent être considérés comme définitivement acquis: Tout d'abord il est hors de doute après l'examen fait par M. Cowley des originaux et pour quiconque veut bien examiner le fac-similé publié dans le Journal que l'écriture des documents épistolaires trouvés par M. M. A. Stein est d'origine araméenne, mais qu'elle est d'un type fortement aberrant; d'autre part il reste établi que la langue qui se dissimule sous cette graphie n'est pas un dialecte sémitique; M. Cowley, qui fait autorité en la matière n'y a retrouvé que ce que l'on est convenu d'appeler des "cryptogrammes", c'est à dire de ces mots sémitique fossiles qui étaient écrits mais non lus en pehlvi par exemple et qui faisaient partie de l'écriture araméenne, comme des sortes de sigles. Il a reconnu ainsi de façon à peu près certaine to $m n$ (loc. laud., p. 163) et la négation ל l' (loc. laud., p. 166). Il ne nous a pas été possible non plus de découvrir dans la langue "inconnue" un parler sémitique, et M. Israël Lévi, qui a bien voulu examiner le fac-similé publié par M. Cowley, n'y a pas réussi davantage. Dès lors il y avait deux possibilités dont il fallait tenir compte avant tout, et d'ailleurs de façon inégale: celle que la langue en question fût turque ou iranienne. Déjà M. Cowley avait été amené tout naturellement après avoir trouvé des "cryptogrammes" à l'hypothèse qu'il avait à faire à du pehlvi : suivant cette idée, il avait cru reconnaître הותאה $x \bar{u} t \bar{a} \bar{\imath}$ (loc. laud., p. 164) et T' يك yäk (loc. laud., p. 166) c'est à dire les mots "maître, seigneur", et "un" précisément sous leur forme persane la plus caractéristique: 
ailleurs qu'en Perse le nom de nombre "un" et le mot "maître" affectent en effet des formes tout différentes. On aperçoit de suite l'importance de pareilles identifications: si elles étaient exactes les lettres retrouvées par M. M. A. Stein nous fournissaient les spécimens les plus anciens que nous possédions du pehlvi des livres, et nous attestaient l'usage de cette forme particulière du moyen persan en Chine, à l'est de 'Tourfan, d'où les expéditions allemandes avaient rapporté les précieux documents qui témoignent qu'au $3^{\mathrm{e}}$ siècle de notre ère la sourde intervocalique $t$ de הותא par exemple était déjà devenue la sonore $d$. Malheureusement les cryptogrammes si nombreux du pehlvi des livres restaient impossibles à retrouver; les graphies sémitiques des conjonctions, des pronoms, et des verbes demeuraient méconnaissables bien qu'elles fussent connues par ailleurs. En fait cette amorce de solution aboutissait à ajouter de nouveaux mystères aux anciens.

D'autre part l'examen de l'alphabet que M. Cowley avait dressé, à titre d'hypothèse bien entendu, mais tel qu'il résultait en fin de compte de la somme des identifications qu'il avait cru pouvoir faire révélait deux lacunes singulières: on n'y trouvait de I que final et point de $i$. Or $n$ et $z$ existent l'un et l'autre en turc comme en iranien (cf. loc. laud., p. 166) et sont, on le sait, très abondants en pehlvi. En revanche le second des deux caractères que M. Cowley lisait , celui auquel il joignait d'ailleurs un point d'interrogation, devait être selon toute vraisemblance un J: au point de vue paléographique il apparaît sur le fac-similé et dans les diverses reproductions que $\mathbf{M}$. Cowley a données comme fort voisin du nūn araméen et $M$. Israël Lévi auquel cette hypothèse a été communiquée de suite l'a couverte de son autorité en s'y ralliant aussitôt. Il y a là, au premier abord, une observation intéressante et une probabilité ; à y regarder de près au contraire, un changement grave dans la lecture de M. Cowley tout entière et une quasi certitude. 
500 LA LANGUE ET L'ECRITURE INCONNUES

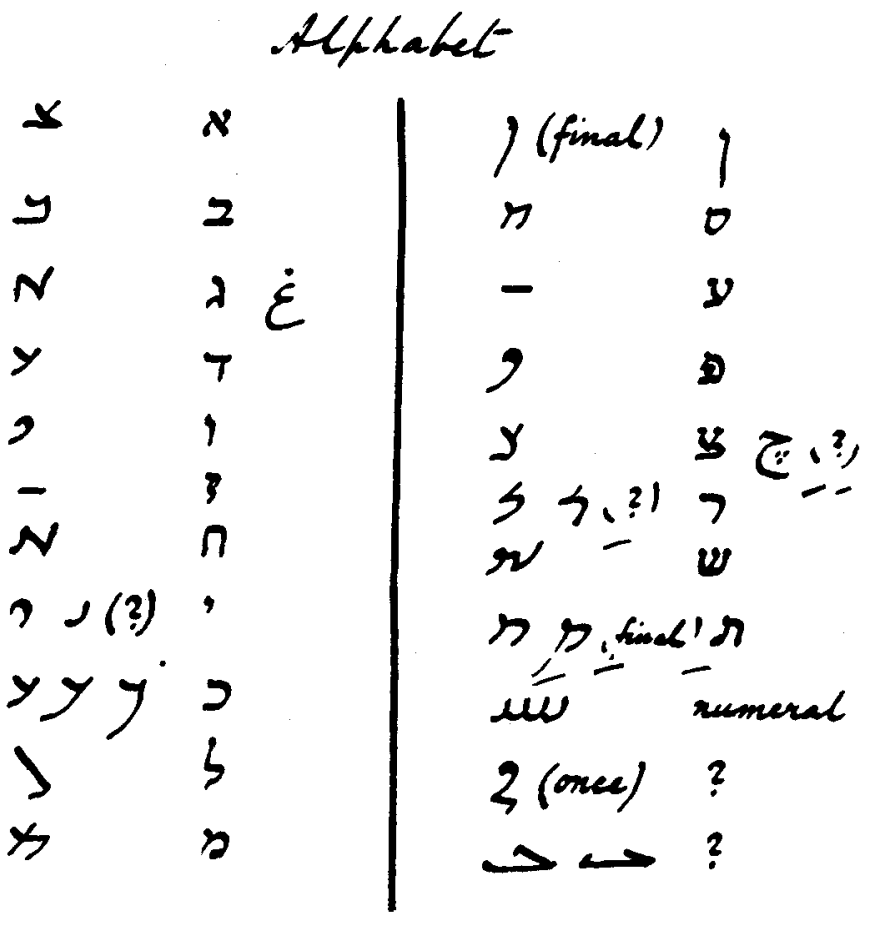

Address

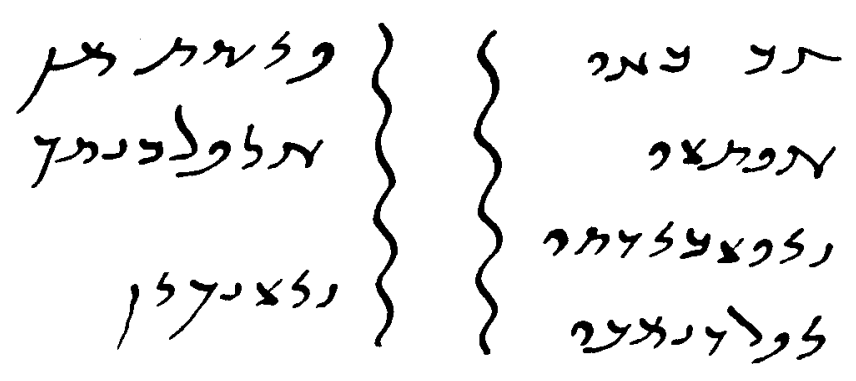

Downloaded from https://www.cambridge.org/core. INSEAD, on 25 May 2018 at 04:25:39, subject to the Cambridge Core terms of use, available at https://www.cambridge.org/core/terms. https://doi.org/10.1017/S0035869X00041575 
En effet en substituant simplement la valeur de $n$ à celle de $y$ on obtient de suite une indication précieuse sur la nature de la langue des documents en question et sur la direction dans laquelle il convient de chercher la clef de l'écriture. Dans l'adresse même, dans la partie de gauche où figure le nom de l'expéditeur, on lit à la deuxième ligne le mot attendu (cf. loc. laud., p. 163) de "serviteur" sous sa forme sogdienne de בנתך qui se trouve répétée fréquemment dans les documents bouddhiques en écriture sogdienne, et qui apparaît aussi sans l'élargissement en -k-dans les textes chrétiens publiés par M. F. W. K. Müller (Sitzungsberichte de l'Académie de Berlin, 1907, pp. 264-7). C'est là une forme nettement dialectale qui s'oppose au bandak du pehlvi (pers. بند) et au $b a^{n} d a k a$ du vieux perse. Le mot correspondant du côté droit de l'adresse, le mot " seigneur" se trouve aussitôt confirmé : seulement c'est le sogdien חות qu'il faut lire, qui dans les textes bouddhiques en écriture sogdienne est $\gamma w t^{\prime} w$, prononcé xutaw, selon la supposition faite par M. Andreas et moi dès janvier, et non le 'חות suspect qu'y avait cru reconnaître M. Cowley, préoccupé de retrouver le correspondant du pehlvi דותאה et du persan خدائ (v. loc. laud., p. 164). Du coup se trouve enfin éliminée la distinction sûrement artificielle, tant elle était ténue et incertaine, que M. Cowley avait été amené à établir entre deux variétés indéfinissables et interchangeables d'un même caractère qui est en fait un $w$, I.

Ainsi l'on se trouve en présence de cette conclusion, provisoire encore, mais qui va se vérifier avec une rapidité singulière que les lettres rapportées par M. M. A. Stein des confins de la Chine propre et étudiées par M. Cowley sont en langue sogdienne notée au moyen d'un système graphique qui est à peu près à celui des manichéens et des chrétiens de langue sogdienne ce que le pehlvi est au pazend. Or il importe de noter avant tout qu'une écriture à cryptogrammes sémitiques a existé pour le sogdien JRAS. 1911. 
comme pour le persan, avec cette différence que le nombre des "sigles" étrangers y était singulièrement moindre ; c'est dans cette écriture que sont fixés les textes bouddhiques que les expéditions en Asie centrale nous ont révélés et dont des spécimens précieux se trouvent à Berlin, à Londres, et surtout peut-être à Paris ; c'est dans cette écriture qu'est rédigée l'inscription sogdienne de Qara-Balgassoun, où M. F. W. K. Müller a reconnu dès 1909 un texte iranien parsemé de cryptogrammes (Sitzungsberichte de l'Académie de Berlin, 1907, p. 726 et suiv., et surtout p. 729). Il n'y a donc rien de surprenant. à retrouver dans les documents de M. M. A. Stein les בנתך termes sémitiques et : c'est au contraire une constatation qui s'accorde avec les données historiques et linguistiques connues, et qui est conforme aux probabilités.

C'est en outre une observation essentielle au point de vue de l'écriture. L'usage des cryptogrammes est particulier, comme nous venons de l'indiquer, non pas à un dialecte ou à un peuple, mais à un système graphique; il appartient en commun aux textes bouddhiques les plus anciens et à l'inscription manichéenne de Qara-Balgassoun. Si les textes manichéens déchiffrés et publiés par M. F. W. K. Müller avec la maîtrise que l'on sait n'offrent pas de cryptogrammes, c'est qu'ils sont notés en écriture estranghélo modifiée dite manichéenne. La conclusion s'impose: les textes épistolaires dont il s'agit ici doivent être en écriture sogdienne, s'ils sont vraiment en langue sogdienne avec cryptogrammes; ou plutôt, conformément aux conclusions d'une étude dont les principaux résultats ont été communiqués à la Société Asiatique de Paris à la séance du 13 janvier 1911, et qui est actuellement sous presse, ils doivent être notés en une cursive araméenne intermédiaire entre l'araméen proprement dit et l'écriture sogdienne d'où est issu l'alphabet ouigour. Il ne faut done pas essayer de les lire en partant des systèmes graphiques des langues 
sémitiques, ni surtout en s'inspirant de leur évolution propre et du sens dans lequel ils se sont développés; c'est du point d'arrivée, l'écriture sogdienne, qu'il faut partir en remontant.

A procéder ainsi la ressemblance entre l'écriture du facsimilé publié par M. Cowley et celle des plus beaux textes bouddhiques sogdiens d'Asie centrale apparaît tout à coup à qui a pratiqué suffisamment ces derniers et a étudié de près leur graphie et leur ductus. Les quelques ligatures encore rares, du document dû̀ à M. M. A. Stein sont particulièrement claires: 1 par exemple montre déjà la liaison du avec le ; suivant par le trait d'en haut, et non de gauche, comme cela se fait en syriaque. Les ligatures du $ת$ avec le 1 ou le $J$ qui suivent annoncent déjà le ductus sogdien; de même celles du $\boldsymbol{E}$ et du I avec le 1 . La forme du $\supset$, celle du $\supset$ sont déjà toutes proches de celles du $p$ et du $k$ sogdiens; celle du $\boldsymbol{N}$ est quasi identique. Il y a plus: on sait que, sauf quelques exceptions le $J$ et le $i$ sont confondus en écriture sogdienne: si l'on admet une confusion pareille dans le document publié par M. Cowley on retrouve aussitôt le cryptogramme 7 qui vient remplacer de façon toute naturelle et singulièrement avantageuse ce $T^{\prime}$ purement persan que $M$. Cowley avait été obligé d'admettre et qui ne pouvait trouver place en un document sogdien. L'on sait que ce $\rceil^{i}$ est le cryptogramme par excellence et que son absence suffirait presque à faire douter du caractère "pehlvi" ou "cryptographique" d'un texte (cf. loc. laud., p. 166).

Voici d'autres conséquences plus graves qui aboutissent toutes à des résultats importants. Après les changements de lecture qui ont été admis jusqu'ici, il se trouve que si le $J$ et $\mathbf{i}$ sont représentés, le ' en revanche ne l'est plus. D'après la direction générale de l'évolution des écritures sémitiques on s'attend d'ailleurs à le voir noté par un signe de dimensions extrêmement restreintes; et il n'y a plus de caractère réduit à tel point dans le système 
graphique nouveau que M. Cowley met sous nos yeux. Mais l'étude de l'alphabet sogdien nous apprend què dans I'Tran du nord le 'a suivi un développement différent: il ne s'est pas rabougri comme en hébreu, en syriaque, en palmyrénien, il a au contraire grandi. Il y est représenté par un trait oblique large et fort qui ressemble assez à un $\$$ syriaque tronqué et se termine par une petite barre disposée verticalement par rapport à la haste principale. Bref, il est tout pareil dans son ensemble au caractère nouveau que M. Cowley a lu ר. Quant à celuici, qui maintenant fait défaut, il se retrouve lui aussi si l'on remonte de l'écriture sogdienne bouddhique à celle des lettres en question au lieu de chercher à reconnaître en celle-ci les caractères sémitiques: il est représenté par le signe où $M$. Cowley a cru voir un 7 , qui ressemble en effet au $\supset$ de façon exceptionnelle et ne diffère de lui que par la dimension de sa queue, exactement comme le fait le $r$ par rapport au $k$ sogdien bouddhique. Et, de fait, si l'on substitue ces valeurs nouvelles aux anciennes, celle de ' à celle de 7 , et celle de 7 à celle de 7 , tout en tenant compte de ce qui a été dit plus haut du $\mathrm{J}$ et $d u \quad i$, on voit l'aspect du texte se modifier singulièrement, se préciser et gagner en clarté; ainsi on lit immédiateאחדיר אחיר et (v. loc. laud., p. 165) les beaux cryptogrammes

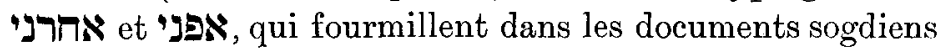
bouddhiques et qui sont formés des conjonctions sémitiques אחר suivies de leur traduction ' (cf. p. ex. Muiller, Sitzungsberichte de l'Académie de Berlin, 1909, pp. 727-8). Ces identifications sont d'une importance toute particulière, non seulement parce qu'elles portent sur des mots sogdiens bien établis pour la forme comme pour le sens, mais encore parce qu'elles confirment l'un des traits les plus caractéristiques de la langue des textes étudiés ici. M. Cowley a fait ressortir très justement que ces mots, qui restaient inintelligibles pour lui, 
étaient munis souvent, comme le sont les conjonctions en pehlvi, d'affixes qui devaient être sans doute les formes enclitiques des pronoms personnels, $\Delta^{\circ}$ pour la première, $\Omega^{\circ}$ et $j \pi^{\circ}$ pour la seconde, $\mathbb{W}^{\circ}$ pour la troisième personne; or, ce sont bien là des formes nettement iraniennes et sogdiennes, comme, par exemple, $\boldsymbol{W}^{\circ}$, qui est particulièrement fréquent et alterne avec " $\mathscr{*}$. De la même façon on arrive à lire dans l'adresse בי "something written" au lieu de פרשת "something sent" (loc. Jaud., p. 164).

Un dernier point à éclaircir, pour compléter l'alphabet nouveau, est celui de la notation du 7 , dont la place est vide momentanément, et qui est un son relativement fréquent en sogdien. Nous n'hésitons pas à admettre que, comme dans l'écriture sogdienne proprement dite, c'est le caractère qui vaut $\zeta$ dans les mots sémitiques, dans les cryptogrammes, qui sert à noter 7 dans les mots sogdiens. Ceci nous permet d'ailleurs immédiatement de lire le début de la partie gauche de l'adresse qui devient: פישת מן חיפר בנתך c'est à dire pyšt $M N$ hyp $\beta$ Bntk, en transcrivant lettre pour lettre et en notant les cryptogrammes par des majuscules, ce qui signifie sans doute "écrit de son serviteur . . ." " car hyp $\delta^{1}$ qui était prononcé à peu près xēpa $\theta$ est la forme correcte du réfléchi en sogdien où il signifie, placé devant les noms, ce que veulent dire en français "son, sa, ses". De la même façon on arrive à lire כד "si, quand" le mot où M. Cowley voyait בל cryptogramme du sens de "tout" (loc. laud., p. 166); on retrouve la forme sogdienne "אח du verbe "aller" aux lignes 2 et 4 du fac-similé, celles de בירת de "tenir, obtenir" aux lignes 2 et 5, et חרית de "acheter" à la ligne 5 et d'autres encore. Surtout, il est intéressant de constater que la ligne $1 \mathrm{du}$ document, qui contient la formule d'introduction devient

1 Nous avons maiutenu ici, comme on le voit, la distinction faite par M. Cowley entre 7 et $\gamma$, quoiqu'elle nous paraisse très ténue et peut-être bien artificielle. 
tout à fait intelligible ou peu s'en faut, sauf la part à faire aux noms propres possibles. Le destinataire est introduit

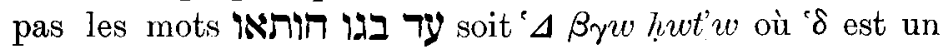
cryptogramme reconnu par M. Cowley (loc. laud., p. 163) mais faussement transcrit par vad selon une tradition qui croit devoir lire un $Y$ là où il y a en réalité un $y$ cursif et rattaché à la lettre qui se trouve à sa gauche, où $\beta \gamma w$ est tout comme hwot'w une belle forme sogdienne attestée dans les textes bouddhiques comme aussi sur le monument de Qara-Balgassoun (cf. F. W. K. Müller, Sitzungsberichte de l'Académie de Berlin, 1909, p. 728). Suit l'indication de l'expéditeur dans les mêmes termes que sur l'adresse et qu'il est inutile de répéter ici. En fin vient la formule de salutation qui est très intéressante. Elle se termine en effet par un mot, jusqu'ici inintelligible, mais qui n'est autre que I'צr' נמצ' $n$ 'cyw, c'est à dire un terme sogdien bien connu qui signifie dans les textes bouddhiques où il apparaît fréquemment "hommage, adoration" et qui traduit ici tout simplement le cryptogramme sémitique של qui le précède. Devant celui-ci enfin est un nom de nombre où M. Cowley a reconnu, non sans hésitation (loc. laud., p. 164) une forme altérée du sémitique ô "mille"; il lisait, en effet, לך", ce que, d'après les conclusions exposées plus haut, on doit lire נלר. Or, tandis que פ'tait non seulement anormal mais inexplicable et isolé, נלך est précisément la forme du nombre mille en sogdien bouddhique et peut s'expliquer comme une altération cursive de אל sous les doigts d'hommes qui ne prononçaient plus rien qui fût en rapport avec ce qu'ils notaient et qui ne pouvaient plus voir qu'un chiffre dans ce qui avait été un mot. En effet le passage de $\mathbf{N}$ a' est inexplicable, et presque impossible dans l'écriture sogdienne des documents bouddhiques aussi bien que dans la cursive des lettres rapportées par M. M. A. Stein ; au contraire le changement de $\mathbf{N}$ en s'explique dans l'un et l'autre système graphique par une simple oblitération et par la perte d'un petit trait 
transversal planté sur le principal. Il est à noter que la même altération s'est produite précisément dans l'alphabet sogdien tardif, en ouïgour, où, sauf des cas déterminés, l'n ne se distingue plus de l' $\alpha$. On voit comment dans le détail le rapprochement se confirme entre la langue et l'écriture sogdiennes que nous connaissions déjà et celle que les découvertes de M. M. A. Stein nous révèlent. 'Tout le système des chiffres est le même dans les documents de l'un et l'autre type: daus la lettre publiée par M. Cowley on trouve les groupes d'unités, les signes pour 10 et pour 20 que l'on connaît déjà par les textes bouddhiques et qui sont d'ailleurs apparentés à ceux du syriaque. 